\title{
GROUND STATES OF CLASSICAL ONE-DIMENSIONAL LATTICE MODELS
}

\author{
Max TEUBNER \\ Max-Planck-Insitut für biophysikalische Chemie, Postfach 2841, W-3400 Göttingen, Germany
}

Received 2 July 1990

Some theorems concerning the ground states of classical one-dimensional lattice models with arbitrary interactions of finite range are proved.

\section{Introduction}

The problem of determining the ground states of classical spin models has received comparatively little attention. This is somewhat surprising since, apart from special cases, this problem may be quite difficult in two or higher dimensions. In fact, the closely related domino problem is algorithmically unsolvable in two dimensions [1]. Contrary to this, the ground states of one-dimensional models with a finite spin space $S$ are in principle well known. This arises from the fact that the period of any ground state for interaction range $r$ is $\leqslant|S|^{r}$. Nevertheless, the number of possible ground states can be quite large even for moderate values of $|S|$ and $r$. For example, the Ising model $(|S|=2)$ with $r=5$ has 30176 possible ground states (see section 11). Therefore, and as an introduction to the much more difficult problem in higher dimensions, it seems meaningful to develop some experience in the onedimensional situation.

Previous work on the problem is quite sparse. Morita and Horiguchi [2] have determined the ground states for the Ising model for $r=2$ and Bundaru et al. [3] for $r=3$. Some general results on systems with inversion symmetry have been obtained by Morita [4]. For $r=\infty$ very interesting new phenomena appear $[5,6]$, but this paper is restricted to finite $r$.

\section{Configurations and correlations}

A configuration $\omega$ is a doubly infinite sequence 


$$
\omega=\ldots \sigma_{-2} \sigma_{-1} \sigma_{0} \sigma_{1} \sigma_{2} \ldots
$$

with $\sigma_{i} \in\{-1,1\}$ such that all the correlations of range $s \leqslant r$

$$
\left\langle\sigma_{i} \sigma_{i+i_{1}} \ldots \sigma_{i+i_{i}} \sigma_{i+s}\right\rangle=\lim _{N \rightarrow \infty} \frac{1}{2 N+1} \sum_{i=-N}^{N} \sigma_{i} \sigma_{i+i_{1}} \ldots \sigma_{i+i_{t}} \sigma_{i+s}
$$

are well defined. Let $\Omega$ be the set of all configurations. Two configurations are equivalent if they differ by a translation. Equivalent configurations have the same correlations (translational invariance).

For $r=0$ the only correlation is the magnetization $\langle\sigma\rangle$. For $r=1$ we have, in addition, the nearest neighbour (NN) correlation $\left\langle\sigma_{i} \sigma_{i+1}\right\rangle$. For $r=2$ there appcar two additional correlations, namely $\left\langle\sigma_{i} \sigma_{i+2}\right\rangle$ and $\left\langle\sigma_{i} \sigma_{i+1} \sigma_{i+2}\right\rangle$. In general, proceeding from $r-1$ to $r, 2^{r-1}$ new correlations appear. These are in a one-to-one correspondence with the $2^{r-1}$ subsets of the $r-1$ spins $\sigma_{i+1} \ldots \sigma_{i+r-1}$. Therefore, the total number of correlations relevant to the interaction range $r$ is $2^{r}$.

\section{The correlation polyhedron $\mathbf{P}_{r}$}

When plotted as points in a space of $2^{r}$ dimensions, every configuration $\omega$ generates a point $\langle\boldsymbol{\sigma}\rangle=\langle\boldsymbol{\sigma}(\omega)\rangle$ in this space. Let $\mathrm{P}_{r}$ be the set of points obtained in this way. Since

$$
\left|\left\langle\sigma_{i} \ldots \sigma_{i+r}\right\rangle\right| \leqslant 1
$$

$\mathbf{P}_{r}$ is contained in the $2^{r}$-dimensional hypercube $[-1,1]^{2^{r}}$. It is easy to sec that $\overline{\mathrm{P}}_{r}$ (the closure of $\mathrm{P}_{r}$ ) is a convex set (see appendix).

Let

$$
H=\sum_{s=1}^{r} \sum E_{i_{1} \ldots i_{t}}^{(s)}\left\langle\sigma_{i} \sigma_{i+i_{1}} \ldots \sigma_{t+t_{i}} \sigma_{i+s}\right\rangle
$$

be a Hamiltonian. Combining the $E \ldots$ and the correlations into $2^{r}$-dimensional vectors $\boldsymbol{E}$ and $\langle\boldsymbol{\sigma}\rangle$, respectively, the ground states of $H$ are the minimizers of

$$
\min \left\{\boldsymbol{E} \cdot\langle\boldsymbol{\sigma}\rangle:\langle\boldsymbol{\sigma}\rangle \in \mathrm{P}_{r}\right\}
$$

This means geometrically that the ground states are just the extreme points of the convex set $P_{r}$. Since the number of ground states in one dimension is 
known to be finite, $\mathrm{P}_{r}$ is a polyhedron whose vertices correspond to the possible ground states [7].

Example. $r=1$. This is the well known Ising model with NN interactions. There are $2^{1}=2$ correlations, namely $\langle\sigma\rangle$ and $\left\langle\sigma_{i} \sigma_{i+1}\right\rangle$, and three ground states: $(+)_{p},(-)_{p}$ and $(+-)_{p}$ (see fig. 1$)$. (By $\left(\sigma_{1} \ldots \sigma_{n}\right)_{p}$ we denote the periodic configuration $\left.\ldots \sigma_{1} \ldots \sigma_{n} \sigma_{1} \ldots \sigma_{n} \ldots\right)$.

The polyhedron possesses the following elementary properties:

(1) Spin inversion induces a reflection that maps $P_{r}$ onto itself. It inverts the sign of a correlation with an odd number of spins and leaves invariant the even ones. In fig. 1 this is the reflection symmetry $\langle\sigma\rangle \rightarrow-\langle\sigma\rangle,\left\langle\sigma_{i} \sigma_{i+1}\right\rangle \rightarrow$ $\left\langle\sigma_{i} \sigma_{i+1}\right\rangle$.

(2) The mapping

$$
\ldots \sigma_{-2} \sigma_{-1} \sigma_{0} \sigma_{1} \sigma_{2} \ldots \rightarrow \ldots \sigma_{2} \sigma_{1} \sigma_{0} \sigma_{-1} \sigma_{-2} \ldots
$$

maps every correlation on to its mirror image. This global symmetry comes into play for $r \geqslant 3$ (mapping $\left\langle\sigma_{i} \sigma_{i+1} \sigma_{i+3}\right\rangle$ onto $\left\langle\sigma_{i} \sigma_{i+2} \sigma_{i+3}\right\rangle$ ).

(3) The projection of $\mathrm{P}_{r+1}$ on the space spanned by all the correlations of range less than or equal to $r$ is just $\mathbf{P}_{r}$. For example, projecting $\mathbf{P}_{1}$ in fig. 1 on the $\langle\sigma\rangle$-axis yields the segment $[-1,1]$ with the two ferromagnetic ground states as vertices.

(4) The origin $\langle\boldsymbol{\sigma}\rangle=\boldsymbol{\theta}$ is contained in the interior of $\mathbf{P}_{r}$.

Property (4) stems from the fact that any random configuration is mapped onto the origin. Any other point in $\mathrm{P}_{r}$ therefore corresponds to non-random

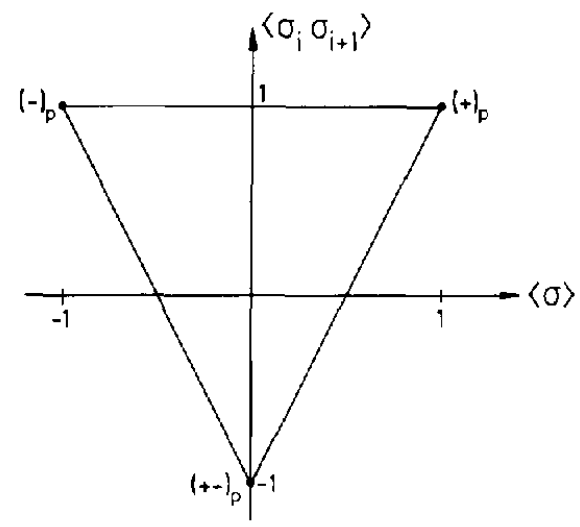

Fig. 1. For $r=1$ the correlation polyhedron is a triangle with vertices $(1,1),(-1,1)$ and $(0,-1)$ corresponding to the ferromagnetic states $(+)_{p}$ and $(-)_{p}$ and to the antiferromagnetic state $(+-)_{p}$, respectively. 
configurations. This suggests that the entropy of a configuration may be determined by its position in $\mathrm{P}_{r}$. This, in fact, is true quite generally. It stems from the fact that the entropy can be expressed either by the fields (the $E_{i_{1}} \ldots i_{t}$ in eq. (3)) or by their conjugate densities (the $\left.\left\langle\sigma_{i} \sigma_{i+i_{1}} \ldots \sigma_{i+i_{r}} \sigma_{i+s}\right\rangle\right)$.

\section{The graph $G_{r}$}

Van Aardenne-Ehrenfest and de Bruijn [8] (see also ref. [9]) and independently Bundaru et al. [3] have introduced a useful representation of the ground states in terms of a graph $\mathrm{G}_{r}$ (see fig. 2). Consider a graph with $2^{r}$ nodes $\sigma_{1} \ldots \sigma_{r}$. Two nodes $A=\sigma_{1} \ldots \sigma_{r}$ and $A^{\prime}=\sigma_{1}^{\prime} \ldots \sigma_{r}^{\prime}$ are connected by an arrow, if and only if the last $r-1$ spins of $A$ are identical to the first $r-1$ spins of $B$. Every configuration can be represented by an infinite path in this graph, and the ground states are in a one-to-one correspondence with the closed paths on $G_{r}$ without self-intersections. In this way Bundaru has identified the 19 ground states for $r=3$ (for $r=2$ there are the six ground states $(+)_{p},(-)_{p}$. $\left.(+-)_{p},(++-)_{p},(--+)_{p},(++--)_{p}\right)$. In particular, the period of any ground state is less than or equal to $2^{r}$ and the ground states of maximal period are the Hamiltonian paths in this graph. These states of maximal period are known as de Bruijn states. Their number is $2^{f(r)}$ with $f(r)=2^{r-1}-r$.

\section{The densities $\boldsymbol{n}_{\sigma}$}

Consider a configuration $\omega$ and let

$$
n_{\boldsymbol{\sigma}}^{(r)}(\omega)=n_{\sigma_{1} \ldots r_{r+1}}^{(r)}
$$

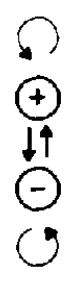

$r=1$

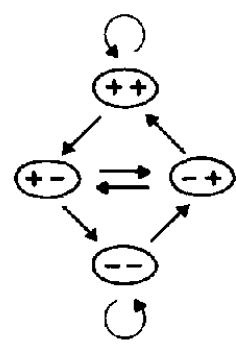

$r=2$

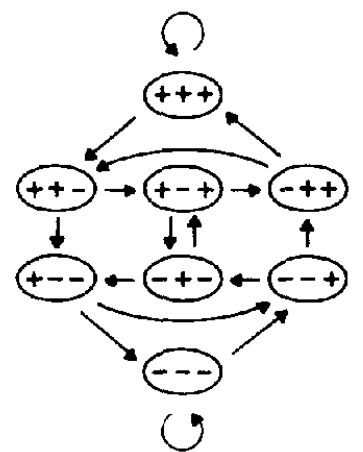

$r=3$

Fig. 2. Graphs $G_{\text {, for }} r=1,2,3$. 
be the density of the chain $\sigma_{1} \ldots \sigma_{r+1}$ in $\omega$. For example, the antiferromagnetic ground state $(+-)_{\mathrm{p}}$ has $n_{++}^{(1)}=n_{--}^{(1)}=0, n_{+-}^{(1)}=n_{-+}^{(1)}=\frac{1}{2}$. The $n_{\sigma}$ are non-negative,

$$
n_{\sigma}^{(r)} \geqslant 0
$$

and normalized,

$$
\sum_{\boldsymbol{\sigma}} n_{\boldsymbol{\sigma}}^{(r)}=1
$$

Furthermore, they satisfy the following linear relations:

$$
n_{\sigma_{1} \ldots \sigma_{r^{+}}}^{(r)}+n_{\sigma_{1} \ldots \sigma_{r^{-}}}^{(r)}=n_{+\sigma_{1} \ldots \sigma_{r}}^{(r)}+n_{-\sigma_{1} \ldots \sigma_{r}}^{(r)} \quad\left(=n_{\sigma_{1} \ldots \sigma_{r}}^{(r-1)}\right),
$$

which expresses the fact that the next spin to the right or left is either + or - . The $n_{\sigma}^{(r)}$ can be expressed in terms of the correlations

$$
n_{\tau_{1} \ldots \tau_{r+1}}^{(r)}=2^{-r-1}\left\langle\left(1+\tau_{1} \sigma_{i}\right)\left(1+\tau_{2} \sigma_{i+1}\right) \ldots\left(1+\tau_{r+1} \sigma_{i+r}\right)\right\rangle .
$$

Since there are $2^{r}$ independent correlations of range $r$, the number of independent $n_{\sigma}^{(r)}$ is also $2^{r}$. A state can be characterized either by the correlations or by the densities $n_{\sigma}$. For $r=1$, e.g., we have the four densities $n_{\tau_{1} \tau_{2}}^{(1)}$ with normalization

$$
n_{++}^{(1)}+n_{+-}^{(1)}+n_{-+}^{(1)}+n_{--}^{(1)}=1
$$

and the linear relation

$$
n_{+-}^{(1)}=n_{-+}^{(1)} .
$$

Now consider the graph $\mathrm{G}_{r}$. An arrow in this graph is characterized by an $(r+1)$-tuple $\sigma_{1} \ldots \sigma_{r} \sigma_{r+1}$ from the node $\left(\sigma_{1} \ldots \sigma_{r}\right)$ to the next node $\left(\sigma_{2} \ldots \sigma_{r} \sigma_{r+1}\right)$. It is clear that the multiplicities with which these arrows are traversed by a closed path in $\mathrm{G}_{r}$ are identical to the $n_{\sigma}^{(r)}$ of the corresponding configuration. Similarly, the multiplicities with which the nodes are traversed are identical to the corresponding $n_{\sigma}^{(r-1)}$. Relation (6) is a conservation law for the associated flow. For a ground state of period $p$, the $n_{\sigma}^{(r)}$ are either 0 or $1 / p$ and the same is true for the $n_{\sigma}^{(r-1)}$.

In particular, for a maximal ground state, where each node is visited precisely once, all the $n_{\sigma}^{(r-1)}=1 / p=2^{-r}$. It follows from (7) that all the correlations of range less than or equal to $r-1$ vanish for maximal ground states. 


\section{The faces of $\mathbf{P}_{r}$}

Our aim is to obain a characterization of the ground states, their number, period, ctc. Now a polyhedron can be characterized either by its vertices or, dually, by its faces. While a vertex corresponds to a ground state, a face corresponds to a Hamiltonian having a maximal number of coexisting phases. In a sense, the faces are more fundamental than the vertices. Firstly, their number is much smaller $\left(\sim 2^{r+1}\right.$ compared with the number of vertices, which is $\sim 2^{2^{r^{-1}}}$ ). Secondly, in contrast to the vertices, it turns out that the faces can be easily and explicitly enumerated. From a practical viewpoint, knowledge of the faces permits the determination of the ground state of any particular Hamiltonian by the techniques of linear programming.

A face corresponds analytically to a linear inequality for the correlations, and the task consists in finding the complete set of these inequalities. Now a particular kind of linear inequality is

$$
0 \leqslant\left\langle\left(1+\tau_{1} \sigma_{i}\right)\left(1+\tau_{2} \sigma_{i+1}\right) \ldots\left(1+\tau_{r+1} \sigma_{i+r}\right)\right\rangle \equiv 1+F_{\tau} \cdot\langle\boldsymbol{\sigma}\rangle .
$$

This means that the density $n_{\tau}^{(r)}$ is non-negative. For example, for $r=1$ we have the following three inequalities:

$$
\begin{aligned}
& F_{++}=2\langle\sigma\rangle+\left\langle\sigma_{i} \sigma_{i+1}\right\rangle \geqslant-1, \\
& F \quad=-2\langle\sigma\rangle+\left\langle\sigma_{i} \sigma_{i+1}\right\rangle \geqslant-1, \\
& F_{--}=F_{--}=-\left\langle\sigma_{i} \sigma_{i+1}\right\rangle \geqslant-1 .
\end{aligned}
$$

Comparing with fig. 1 we observe that we have already found all the linear inequalities. Thus, for $r=1$ the set (10) is complete. The following theorem states that this is true in general.

Theorem 1. The number of faces of $P_{r}$ is 3 for $r=1$ and $2^{r+1}-2$ for $r>1 . \bar{P}_{r}$ is the intersection of the half-spaces

$$
F_{\tau_{1} \ldots \tau_{t+1}} \cdot\langle\boldsymbol{\sigma}\rangle \geqslant-1 .
$$

All the $F_{\tau_{1} \ldots \tau_{r+1}}$ are different with the exceptions

$$
\begin{aligned}
& F_{+\ldots-\cdots-}=F_{-\ldots-+}, \\
& F_{-+\cdots+}=F_{-\ldots+} .
\end{aligned}
$$


Proof. We first prove the last part. Let $F_{\tau_{1} \ldots \tau_{r+1}} \equiv F_{\tau_{i} \ldots \tau_{r+1}}$. Comparing $\left\langle\sigma_{i} \sigma_{i+r}\right\rangle$ we obtain $\tau_{1} \tau_{r+1}=\tau_{1}^{\prime} \tau_{r+1}^{\prime}$. Comparing $\left\langle\sigma_{i} \sigma_{i+k} \sigma_{i+r}\right\rangle$ we find $\tau_{1} \tau_{k+1} \tau_{r+1}=\tau_{1}^{\prime} \tau_{k+1}^{\prime} \tau_{r+1}^{\prime}$, i.e. $\tau_{k}=\tau_{k}^{\prime}$ for $k=2, \ldots, r$. If $\tau_{1}=\tau_{1}^{\prime}$ then $\tau=\tau^{\prime}$. Therefore, let $\tau_{1}=-\tau_{1}^{\prime}$. Then $\tau_{r+1}=-\tau_{r+1}^{\prime}$. Comparing $\langle\sigma\rangle$ we find that $\tau_{1}+\tau_{r+1}=0$. Comparing $\left\langle\sigma_{i} \sigma_{i+k}\right\rangle$ we find $\tau_{k+1}=\tau_{r+1-k}$ for $1 \leqslant k \leqslant r-1$. Comparing $\left\langle\sigma_{i} \sigma_{i+1} \sigma_{i+k}\right\rangle$ we find $\tau_{2} \tau_{k+1}=\tau_{r+1-k} \tau_{r+2-k}$, i.e. $\tau_{2}=\tau_{2+r-k}$ for $2 \leqslant k \leqslant r-1$, i.e. $\tau_{2}=\tau_{3}=\cdots=\tau_{r}$. Therefore, all the $F_{\tau}$ are different with the stated exceptions and their number is $2^{r+1}-2$ for $r>1$.

The rest of the theorem follows from

Theorem 2. Let $n_{\sigma}^{(r)}=n_{\sigma_{1} \ldots \sigma_{r 11}}^{(r)}$ be a set of $2^{r-1}$ non-negative numbers with the following properties:

(a) they are normalized, eq. (5)

(b) they satisfy the conservation laws (6).

Then, for any $\epsilon>0$, there is a periodic configuration $\omega$ with densities $n_{\sigma}^{(r)}(\omega)$ such that

$$
\sum_{\sigma}\left|n_{\sigma}^{(r)}(\omega)-n_{\sigma}^{(r)}\right|<\epsilon .
$$

Proof. We try to interpret the $n_{\sigma}^{(r)}$ as the multiplicities of a configuration in the graph $\mathrm{G}_{r}$ as discussed in section 5. Let the $n_{\sigma_{1} \ldots \sigma_{r}}^{(r-1)}$ be as in eq. (6). All the nodes $\left(\sigma_{1} \ldots \sigma_{r}\right)$ in $\mathrm{G}_{r}$ with $\left.n_{\sigma_{1} \ldots \sigma_{r}}^{(r} \quad 1\right)>0$ form a subgraph $\mathrm{G}_{r_{r}^{\prime}}^{\prime}$. If the theorem has been proved for the connected components of $\mathrm{G}_{r}^{\prime}$, an argument similar to the appendix proves the theorem in the general case. Therefore, we may suppose that $\mathrm{G}_{r}^{\prime}$ is connected. It suffices to prove the theorem for rational $n_{\boldsymbol{\sigma}}^{(r)}$. Let

$$
n_{\sigma}^{(r)}=M_{\sigma}^{(r)} / N
$$

with common denominator $N$ and integers $M_{\sigma}^{(r)}$. For every node in $\mathrm{G}_{r}^{\prime}$ we have

$$
M_{\sigma_{1} \ldots \sigma_{r^{+}}}^{(r)}+M_{\sigma_{1} \ldots \sigma_{r-}-}^{(r)}=M_{+\sigma_{1} \ldots \sigma_{r}}^{(r)}+M_{-\sigma_{1} \ldots \sigma_{r}}^{(r)} \equiv M_{\sigma_{1} \ldots \sigma_{r}}^{(r-1)}>0 .
$$

We start from a node $\left(\sigma^{0}\right)$ in $G_{r}^{\prime}$ and make allowed moves (i.e. $M_{\sigma}^{(r)}>0$ ) to other nodes, thereby traversing a path in $\mathrm{G}_{r}^{\prime}$. Every time an arrow is passed, we decrease its $M_{\sigma}^{(r)}$ by one. This path must stop at some node. It is easy to see that the path can only stop at the initial point $\left(\sigma^{0}\right)$, and is therefore closed. If all of $\mathrm{G}_{r}^{\prime}$ is exhausted, we have completed the task. If not, the whole procedure is repeated. In this way we find that $\mathrm{G}_{r}^{\prime}$ is a finite superposition of closed paths:

$$
\mathrm{G}_{r}^{\prime}=\omega_{1} \cup \omega_{2} \cup \ldots \cup \omega_{n} .
$$


Each of these paths corresponds to a periodic configuration, and the argument in the appendix proves that these configurations can be composed to form a configuration with the required properties.

This theorem shows that the natural set of inequalities $n_{\sigma} \geqslant 0$ is already complete. This is a greatly simplying feature of one-dimensional systems and is in general wrong in higher dimensions. An equivalent version is the following:

Theorem 3. Let

$$
a_{0}+\sum_{1}^{r+1} a_{i} \sigma_{i}+\sum_{i, j=1}^{r+1} a_{i j} \sigma_{i} \sigma_{j}+\cdots+a_{12 \ldots r+1} \sigma_{1} \sigma_{2} \ldots \sigma_{r+1} \geqslant 0
$$

be an inequality valid for any chain $\sigma_{1} \ldots \sigma_{r+1}$ of length $r+1$. Then

$$
a_{0}+\left(\sum_{1}^{r+1} a_{j}\right)\langle\sigma\rangle+\sum_{i, j} a_{i j}\left\langle\sigma_{i} \sigma_{j}\right\rangle+\cdots+a_{12 \ldots r+1}\left\langle\sigma_{i} \sigma_{i+1} \cdots \sigma_{i+s}\right\rangle \geqslant 0
$$

and every linear inequality for the correlations is obtained in this way.

\section{Uniqueness of the ground state}

By uniqueness of the ground state we mean that a vertex of $\mathrm{P}_{r}$ does determine its configuration uniquely.

Theorem 4. For any vertex $\langle\boldsymbol{\sigma}\rangle$ of $\mathrm{P}_{r}$ there is, up to a translation, a unique configuration $\omega$ with $\langle\boldsymbol{\sigma}\rangle(\omega)=\langle\boldsymbol{\sigma}\rangle$.

Proof. Let $F_{\tau^{(i)}}, i=1, \ldots, s$ be the faces of $\mathrm{P}_{r}$ that meet at $\langle\boldsymbol{\sigma}\rangle$ and $F_{\tau^{(i)}}$, $i=s+1, \ldots, 2^{r+1}-2$ be the other faces. Then

$$
\begin{array}{ll}
F_{\tau^{(i)}}\langle\boldsymbol{\sigma}\rangle=-1, & i=1, \ldots, s, \\
F_{\boldsymbol{\gamma}^{(i)}}\langle\boldsymbol{\sigma}\rangle>-1, & i=s+1, \ldots, 2^{r+1}-2,
\end{array}
$$

and the rank of the matrix $\left(F_{\tau^{(i)}}\right)_{i=1, \ldots s}$ is $2^{r}$. In any configuration realizing $\langle\boldsymbol{\sigma}\rangle$ the chains $\boldsymbol{\tau}^{(i)}, i=1, \ldots, s$, are forbidden while the chains $\boldsymbol{\tau}^{(i)}, i>s$ are allowed. Take one of the allowed chains, say $\tau=\left(\tau_{1}, \ldots, \tau_{r+1}\right)$. If both $\tau^{\prime}=\left(\tau_{2}, \ldots, \tau_{r+1}+\right)$ and $\tau^{\prime \prime}=\left(\tau_{2}, \ldots, \tau_{r+1}-\right)$ were not allowed, $F_{\tau^{\prime}} \cdot\langle\boldsymbol{\sigma}\rangle=$ -1 and $F_{\tau^{\prime \prime}} \cdot\langle\boldsymbol{\sigma}\rangle=-1$, and therefore $F_{\tau_{2} \ldots \tau_{*+1}} \cdot\langle\boldsymbol{\sigma}\rangle=-1$. However, 
$F_{\tau_{1} \ldots \tau_{r+1}} \cdot\langle\boldsymbol{\sigma}\rangle>-1$ and $F_{\left(-\tau_{1}\right) \ldots \tau_{r-1}} \cdot\langle\boldsymbol{\sigma}\rangle \geqslant-1$. Adding, we find that $F_{\tau_{2} \ldots \tau_{r+1}} \cdot\langle\boldsymbol{\sigma}\rangle>-1$, which is a contradiction. Therefore, every allowed chain can be continued. Uniqueness follows from the fact that different paths in $G_{r}$ correspond to different $\langle\boldsymbol{\sigma}\rangle$ vectors. Indeed, if the $\langle\boldsymbol{\sigma}\rangle$ vectors for two paths are identical, the same is true for their densities $n_{\sigma}$. In particular, both paths visit the same nodes. As in the proof of theorem 2 the subgraph $\mathrm{G}_{r}^{\prime}$ of paths visited can be decomposed into a finite unit of simple closed paths $\omega_{1} \cup \ldots \cup$ $\omega_{n}$. If $n>1,\langle\sigma\rangle$ is in the convex hull of the $\langle\sigma\rangle\left(\omega_{i}\right)$, contrary to the assumption.

\section{Boundary points and surface tension}

Consider the boundary of fig. 1. A configuration $\omega$ maps upon the boundary $(-)_{p},(+-)_{p}$ if and only if $n_{++}=0$. Therefore, any (rational) point on this boundary can be realized. Furthermore, the surface tension between the two phases vanishes. The same is true for the boundary $(+)_{p},(+-)_{p}$. Now consider a point on the $(+)_{p},(-)_{p}-$ boundary. On this boundary $n_{+-}=$ $n_{-+}=0$. Therefore, the only points on this boundary that can be realized are $(+)_{p}$ and $(-)_{p}$. The surface tension between these phases does not vanish. This is a special case of the following:

Theorem 5. Let $v$ and $\boldsymbol{v}^{\prime}$ be two vertices of $\mathrm{P}_{r}$. Then the line segment $\overline{\boldsymbol{v} \boldsymbol{v}^{\prime}}$ belongs to the boundary of $P_{r}$, and there is the following alternative:

(a) The points on $\overline{\boldsymbol{v} v^{\prime}}$ that can be realized by a configuration are everywhere dense on $\overline{\boldsymbol{v} \boldsymbol{v}^{\prime}}$.

(b) Only the endpoints $\boldsymbol{v}$ and $\boldsymbol{v}^{\prime}$ can be realized.

The surface tension between the two ground states is zero in the first case and positive in the second.

Proof. Let $\omega$ and $\omega^{\prime}$ be the paths in $\mathrm{G}_{r}$ corresponding to the two vertices. If we can find a surface $F_{\tau}$ that contains both $\langle\sigma(\omega)\rangle=v$ and $\left\langle\sigma\left(\omega^{\prime}\right)\right\rangle=v^{\prime}$, then $\overline{\boldsymbol{v} \boldsymbol{v}^{\prime}}$ belongs to the boundary. But $F_{+\ldots+}=-1\left(F_{-\ldots}=-1\right)$ contains every vertex with the exceptions of $\left\langle\boldsymbol{\sigma}(+)_{\mathrm{p}}\right\rangle\left(\left\langle\sigma(-)_{\mathrm{p}}\right\rangle\right)$. This proves the first part of the theorem. The alternative is easily proved: if $\omega$ and $\omega^{\prime}$ have at least one node in common, the first case holds while the second case is true, if their intersection is empty.

The theorem excludes situations where only some isolated points on $\overline{v^{\prime}}$ can be realized. This would physically correspond to crystals that combine stoichiometrically in a fixed proportion to form a mixed crystal. This may, of course, happen in higher dimensions. 


\section{Spin inversion}

Spin inversion is the global symmetry $\omega \rightarrow \bar{\omega}$ where $\omega=\ldots \sigma_{-1}, \sigma_{0}, \sigma_{1}, \ldots$ and $\bar{\omega}=\ldots \bar{\sigma}_{-1}, \bar{\sigma}_{0}, \bar{\sigma}_{1}, \ldots$ with $\vec{\sigma}_{i} \equiv-\sigma_{i}$. The correlations involving an even number of spins are invariant under spin inversion. As $\omega$ ranges over the set of all configurations $\Omega$, the even correlations of range less than or equal to $r$ tracc out a polyhedron $\mathrm{P}_{r}^{\text {even }} . \omega$ and $\bar{\omega}$ always correspond to the same point in $\mathrm{P}_{r}^{\text {even }}$. The configurations corresponding to the vertices of $P_{r}^{\text {even }}$ will be called the even ground states of range $r$. (In this sense the two ferromagnetic ground states $(+)$ and $(-)$ are a single even ground state of degeneracy two).

Let $\omega=\ldots \sigma_{-1}, \sigma_{0}, \sigma_{1}, \ldots$ be a configuration in $\Omega$. Then we definc an operator $M: \Omega \rightarrow \Omega$ by

$$
M \omega=\omega^{\prime}=\ldots \sigma_{-1}^{\prime}, \sigma_{0}^{\prime}, \sigma_{1}^{\prime} \ldots \quad \text { and } \sigma_{i}^{\prime}=\sigma_{i} \sigma_{i-1},
$$

i.e. the $i$ th spin of $M \omega$ in the product of the $i$ th and $(i+1)$ th spins of $\omega$. It is easy to see that $M^{-1}$ is in general two valued $(M(\omega)=M(\bar{\omega}))$. It is one valued for those $\omega$ that satisfy $\omega=\bar{\omega}$, i.e. for periodic configurations of the type $\left(\sigma_{1} \ldots \sigma_{n} \bar{\sigma}_{1} \ldots \bar{\sigma}_{n}\right)_{\mathrm{p}}$.

Theorem 6. The operator $M$ induces a natural isomorphism

$$
M^{*}: \mathbf{P}_{r \cdot 1}^{\text {even }} \rightarrow \mathrm{P}_{r}
$$

In particular, if $\omega^{\prime}=\left(\sigma_{1}^{\prime} \ldots \sigma_{n}^{\prime}\right)_{\mathrm{p}}$ is a ground state of $\mathrm{P}_{r}$, then $M^{-1}\left(\omega^{\prime}\right)$ is an even ground state of range $r+1$, and every even ground state of range $r+1$ is obtained in this way. If $\Pi_{1}^{n} \sigma_{i}^{\prime}=-1$, the two inverse images coincide, and $M^{-1} \omega$ has the form $\left(\sigma_{1} \ldots \sigma_{n} \bar{\sigma}_{1} \ldots \bar{\sigma}_{n}\right)_{\Gamma}$. If $\Pi_{1}^{n} \sigma_{i}^{\prime}=1$, there are two inverse images $\omega_{1}, \omega_{2}$ with $\omega_{2}=\bar{\omega}_{1}$.

The proof is easy, and we only indicate the definition of $M^{*}$. From (18) we have $\langle\sigma\rangle^{\prime}=\left\langle\sigma_{i} \sigma_{i-1}\right\rangle,\left\langle\sigma_{i} \sigma_{i+1}\right\rangle^{\prime}=\left\langle\sigma_{i} \sigma_{i+2}\right\rangle$ etc. and we define

$$
M^{*}\left(\left\langle\sigma_{i} \sigma_{i+1}\right\rangle\right)=\langle\sigma\rangle^{\prime}, \quad M^{*}\left(\left\langle\sigma_{i} \sigma_{i+2}\right\rangle\right)=\left\langle\sigma_{i} \sigma_{i+1}\right\rangle, \quad \text { etc. }
$$

\section{Higher spin}

Except for spin inversion, the results remain valid for arbitrary finite spin with minor modifications. For example, eq. (6) reads for finite spin $q$ 


$$
\sum_{s=1}^{q} n_{\sigma_{1} \ldots \sigma_{r} s}=\sum_{s=1}^{q} n_{s \sigma_{1} \ldots \sigma_{r}} .
$$

\section{Open problems and special results}

We finally list two open problems.

(1) What is the number $N_{\mathrm{g}}$ of possible ground states for Hamiltonians of interaction range $r$ ? This number has been determined in the literature for $r \leqslant 3$. The numbers for $r=4$ and $r=5$ have been calculated by a computer (see table I). For comparison the de Bruijn numbers $N_{\mathrm{dB}}=2^{f(r)}, f(r)=2^{r-1}-r$ are also included in table $\mathrm{I}$. The last column shows that

$$
N_{\mathrm{g}} \sim 3 r N_{\mathrm{dB}}
$$

is in a good approximation to $N_{\mathrm{g}}$. It would be interesting, if this relation could be rigorously proved.

(2) What is the number of ground states $N_{\mathrm{g}}^{1,2}$ of Hamiltonians with one- and two-body interactions only? This number is considerably smaller than $N_{\mathrm{g}}$ and also in general unknown. Even the number of faces of the corresponding polyhedron is unknown. The natural conjecture that all inequalities

$$
0 \leqslant a_{0}+b_{1}\langle\sigma\rangle+\sum_{j=1}^{r} a_{j}\left\langle\sigma_{i} \sigma_{i+j}\right\rangle
$$

can be obtained from local inequalities of the type

$$
0 \leqslant a_{0}+\sum_{j=1}^{r+1} b_{j} \sigma_{j}+\sum_{j, k=1}^{r+1} c_{j k} \sigma_{j} \sigma_{k}
$$

is wrong for $r \geqslant 5$. For example, for $r=5$ the inequality

$$
0 \leqslant 4-2\left\langle\sigma_{i} \sigma_{i+1}\right\rangle-5\left\langle\sigma_{i} \sigma_{i+2}\right\rangle+3\left\langle\sigma_{i} \sigma_{i+3}\right\rangle+3\left\langle\sigma_{i} \sigma_{i+4}\right\rangle-3\left\langle\sigma_{i} \sigma_{i+5}\right\rangle
$$

\section{Table I}

\begin{tabular}{lrrl}
\hline$r$ & $N_{\mathrm{g}}$ & $N_{\mathrm{dB}}$ & $N_{\mathrm{g}} / 3 r N_{\mathrm{dB}}$ \\
\hline 1 & 3 & 1 & 1 \\
2 & 6 & 1 & 1 \\
3 & 19 & 2 & 1.06 \\
4 & 179 & 16 & 0.93 \\
5 & 30176 & 2048 & 0.98 \\
\hline
\end{tabular}


is obtained from the (correct) inequality

$$
\begin{aligned}
0 \leqslant & 16-2\left(\sigma_{1} \sigma_{2}-\sigma_{3} \sigma_{4}+\sigma_{5} \sigma_{6}\right)-3\left(\sigma_{2} \sigma_{3}+\sigma_{4} \sigma_{5}\right)-7\left(\sigma_{1} \sigma_{3}+\sigma_{4} \sigma_{6}\right) \\
& -3\left(\sigma_{2} \sigma_{4}+\sigma_{3} \sigma_{5}\right)+4\left(\sigma_{1} \sigma_{4}+\sigma_{2} \sigma_{5}+\sigma_{3} \sigma_{6}\right)+6\left(\sigma_{1} \sigma_{5}+\sigma_{2} \sigma_{6}\right) \\
& -12 \sigma_{1} \sigma_{6}-\sigma_{1} \sigma_{2} \sigma_{3} \sigma_{4}+2 \sigma_{2} \sigma_{3} \sigma_{4} \sigma_{5}-\sigma_{3} \sigma_{4} \sigma_{5} \sigma_{6} \\
& -3\left(\sigma_{1} \sigma_{2} \sigma_{3} \sigma_{5}-\sigma_{2} \sigma_{3} \sigma_{4} \sigma_{6}\right)+3\left(\sigma_{1} \sigma_{3} \sigma_{4} \sigma_{5}-\sigma_{2} \sigma_{4} \sigma_{5} \sigma_{6}\right)
\end{aligned}
$$

where the four-spin correlations cancel on averaging.

Also unknown is the maximal period (the analogue of the de Bruijn states) that can be achieved by a Hamiltonian with one- and two-body interactions only.

For small $r(r \leqslant 6)$ and two-body interactions only some results in this direction are displayed in table II. $N_{\mathrm{g}}^{(2)}$ is the number of possible ground states for Hamiltonians with two-body interactions only,

$$
H=\sum_{j=1}^{r} J_{j}\left\langle\sigma_{i} \sigma_{i+j}\right\rangle
$$

$N_{\text {ineq }}$ is the number of faces of the corresponding polyhedron, i.e. the number of independent inequalities of the type

$$
\sum_{j=1}^{r} a_{j}\left\langle\sigma_{i} \sigma_{i+j}\right\rangle \geqslant-1
$$

and $p_{\max }$ is the maximal period of any ground state. These states of maximal period are all of the form

$$
\left(\sigma_{1} \sigma_{2} \ldots \sigma_{n} \bar{\sigma}_{1} \bar{\sigma}_{2} \ldots \bar{\sigma}_{n}\right)_{\mathrm{p}}
$$

and "type" denotes the sequence $\sigma_{1} \sigma_{2} \ldots \sigma_{n}$.

Table II

\begin{tabular}{lcccl}
\hline$r$ & $N_{\mathrm{g}}^{(2)}$ & $N_{\mathrm{inc:}}$ & $p_{\max }$ & Type \\
\hline 1 & 2 & 2 & 2 & + \\
2 & 3 & 3 & 4 & ++ \\
3 & 5 & 6 & 6 & $1++$ \\
4 & 7 & 12 & 8 & ++++ \\
5 & 15 & 34 & 14 & +++++-+ \\
6 & 27 & 80 & 26 & +++-++-+++-++ \\
\hline
\end{tabular}


We briefly describe how the results have been obtained. For $r \leqslant 3$ the ground states are well known. For $r=4$ it is not difficult to prove that the only additional ground states are $(++++----)_{p}$ and $(++-+---+-)_{p}$. For $r=5,6$ the following "experimental" procedure was adopted:

(1) In order to generate a sufficient set of periodic states as possible candidates for ground states, a stepwise crystal growth model was simulated. The first $r$ spins $\sigma_{1} \sigma_{2} \ldots \sigma_{r}$ and the interaction constants $J_{j}$ were chosen at random. Then the next spin $\sigma_{r+1}$ was adjusted in such a way that $H$ is minimized. This procedure was repeated. After an initial transient a periodic state emerges. This is a possible candidate for a ground state. (It turned out that the majority of these states are indeed ground states).

(2) The pair correlations $\left\langle\sigma_{i} \sigma_{i+j}\right\rangle$ of these states are plotted as points in an $r$-dimensional space and the convex polygon generated by these points is determined. Those states that are not vertices are discarded. The boundary half-planes are the candidates for the inequalities (21).

(3) If all these inequalities can be proved to be correct, the phase diagram is complete, and all the ground states have been found. In order to prove an inequality, it is first rewritten in terms of the $n_{\sigma}^{(r)}$ in the form

$$
\sum_{\sigma} a_{\sigma} n_{\sigma}^{(r)} \geqslant 0
$$

If all the $a_{\sigma} \geqslant 0$, the inequality is valid. Otherwise, (which is the general case) one can try to prove the inequality by employing the relations (6), thereby transforming the $a_{\sigma}$. If, by a series of such transformations, all the $a_{\sigma}$ can be transformed to non-negative quantities, the inequality is proved. This procedure can be programmed on a computer, and in this way all the inequalities for $r=5$ and $r=6$ have been proved, demonstrating the completeness of the ground states.

\section{Appendix. Proof that $\overline{\mathbf{P}}_{r}$ is convex}

Let $x$ and $y^{\prime}$ be two points in $\mathrm{P}_{r}$ and let $\omega, \omega^{\prime}$ be the corresponding configurations. Let $0<\lambda<1$. It suffices to show that for any $\epsilon>0$ there is a configuration $\bar{\omega}$ such that

$$
|\langle\boldsymbol{\sigma}\rangle(\bar{\omega})-\lambda \boldsymbol{x}-(1-\lambda) \boldsymbol{y}|<\epsilon,
$$

where $\langle\boldsymbol{\sigma}\rangle(\bar{\omega})$ is the correlation vector of $\bar{\omega}$. We may assume that $\lambda$ is rational, $\lambda=m / n$. Choose sufficiently large segments $\omega_{N}$ and $\omega_{N}^{\prime}$, such that 


$$
\left|\langle\boldsymbol{\sigma}\rangle\left(\omega_{N}\right)-\boldsymbol{x}\right|<{ }_{3}^{1} \epsilon, \quad\left|\langle\boldsymbol{\sigma}\rangle\left(\omega_{N}^{\prime}\right)-\boldsymbol{y}\right|<\frac{1}{3} \epsilon
$$

Consider the configuration

$$
\begin{gathered}
\bar{\omega}=\ldots \omega_{N} \omega_{N} \ldots \omega_{N} \omega_{N}^{\prime} \omega_{N}^{\prime} \ldots \omega_{N}^{\prime}, \\
m \cdot l \\
n \cdot l
\end{gathered}
$$

where $l$ is an integer. For $l$ sufficiently large $\bar{\omega}$ has the required properties.

The convexity of $\vec{P}_{r}$ is very general, it holds in any number of dimensions and for any spin.

\section{References}

[1] R. Berger, The undecidability of the domino problem, Memoirs, Am. Math. Soc. 66 (1966).

[2] T. Morita and T. Horiguchi, Phys. Lett. A 38 (1972) 223

[3] M. Bundaru, N. Angelescu and G. Nenciu, Phys. Lett. A 43 (1973) 5.

[4] T. Morita, J. Phys. A 7 (1974) 289, 1613.

[5] J. Hubbard, Phys. Rev. B 17 (1978) 494.

[6] P. Bak and R. Bruinsma, Phys. Rev. Lett. 49 (1982) 249.

[7] T. Kudo and S. Katsura, Prog. Theor. Phys. 56 (1976) 435.

[8] T. van Aardenne-Ehrenfest and N.G. de Bruijn, Simon Stevin Wis. Natuurkd. Tijdschr.

[9] S.W. Golomb, Shift Register Sequences (San Francisco, 1967). 